\title{
Metabolic markers, regional adiposity, and adipose cell size: relationship to insulin resistance in African-American as compared to Caucasian women
}

\author{
Candice Allister-Price ${ }^{1,2}$, Colleen M. Craig ${ }^{2}$, Dan Spielman ${ }^{2}$, Samuel S. Cushman ${ }^{3}$, and \\ Tracey L. McLaughlin² \\ ${ }^{1}$ Department of Molecular Biology, School of Veterinary Medicine, University of California, Davis, \\ $\mathrm{CA}$ \\ ${ }^{2}$ Division of Endocrinology, Department of Medicine, Stanford University School of Medicine, \\ Stanford, California \\ ${ }^{3}$ National Institute of Diabetes and Digestive and Kidney Diseases, National Institutes of Health, \\ Bethesda, MD
}

\begin{abstract}
Background/Objectives: African-American women have the greatest prevalence of obesity in the U.S., and higher rates of type 2 diabetes (T2D) than Caucasian women, yet paradoxically lower plasma triglycerides (TG), visceral fat and intrahepatic fat, and higher high-density lipoprotein (HDL)-cholesterol. Visceral fat has not been evaluated against insulin resistance in African-American women, and TG/HDL-cholesterol has been criticized as a poor biomarker for insulin resistance in mixed-sex African-American populations. Adipocyte hypertrophy, reflecting adipocyte dysfunction, predicts insulin resistance in Caucasians, but has not been studied in African-Americans. Our goal was to assess whether traditional correlates of insulin resistance, measures of adiposity and adipocyte characteristics similarly predict peripheral insulin resistance in African-American and Caucasian women.
\end{abstract}

Subjects/Methods: Thirty-four healthy African-American ( $n=17)$ and Caucasian $(n=17)$ women, matched for age (mean $=53.0 \mathrm{yrs}$ ) and BMI (mean $\left.=30 \mathrm{~kg} / \mathrm{m}^{2}\right)$, underwent a steady-state plasma glucose (SSPG) test to measure insulin sensitivity; computed tomography (fat distribution); and a periumbilical scalpel biopsy (adipocyte characterization). By-race analyses utilized ANCOVA; linear regressions evaluated relationships between metabolic/adipose variables. All analyses adjusted for BMI and menopausal status.

Results: Insulin sensitivity did not differ between groups ( $\mathrm{p}=0.65)$. BMI, \%body fat nor thigh fat predicted insulin resistance in African-American women. Fasting TG ( $\mathrm{p}=0.046)$, HDL-cholesterol $(\mathrm{p}=0.0006)$ and TG/HDL-cholesterol ratio $(\mathrm{p}=0.009)$ strongly predicted insulin resistance in

Users may view, print, copy, and download text and data-mine the content in such documents, for the purposes of academic research, subject always to the full Conditions of use:http://www.nature.com/authors/editorial_policies/license.html\#terms

Corresponding author: Candice Allister Price, 1089 Veterinary Medicine Dr. Davis, CA 95616, Phone: 530-752-5379. Fax:

530-754-2474.

Conflicts of Interest

The authors have no conflicts of interest to report. 
African-American women. Despite being lower in African-American women, hepatic fat and VAT correlated with insulin resistance in both groups, as did fasting glucose, VAT/SAT ratio and \%SAT (inverse).

Conclusions: Total adiposity measures and adipocyte hypertrophy did not predict insulin resistance in African-American women, but did in Caucasian women. Plasma TG and HDLcholesterol were significant predictors of insulin resistance in African-American women. Our findings demonstrate the need to identify race and sex-specific biomarkers for metabolic risk profiling.

\section{Keywords}

Steady state plasma glucose test; insulin resistance; African-Americans

\section{Introduction}

African-Americans in the U.S. have the highest prevalence of obesity and the second-highest rate of type 2 diabetes (T2D) after Native Americans/Alaskans (1-3). Several studies have further shown that even independent of obesity, African-Americans (both adults and children) are more insulin resistant than Caucasians (4-6), potentially accounting for the high T2D risk. African-American women, in particular, are at even greater risk for developing T2D, compared to both African-American men and Caucasian women (7). This difference between African-American and Caucasian women has been observed even in spite of African-American women having less visceral fat, lower plasma triglyceride (TG) levels, and higher plasma HDL-cholesterol $(6,8-10)$. These paradoxical phenotypes paired with the elevated risk for T2D support the need for further studies to determine the role of traditional risk factors for insulin resistance in this population, which may shed light on the race-specific pathophysiology of insulin resistance and T2D. Furthermore, insulin kinetics are different in African-Americans vs Caucasians, rendering many measures of insulin resistance inaccurate. Not only is insulin clearance reduced (11), but first phase insulin response is more robust $(5,12)$. These factors could lead to an overestimation of peripheral insulin resistance in this population, as previously pointed out by Pisprasert and colleagues (13), when using methods that rely on insulin concentrations, such as the Homeostatic model assessment (HOMA), Quantitative Insulin Sensitivity Check Index (QUICKI), oral glucose tolerance test (OGTT), and possibly the intravenous glucose tolerance test (IVGTT). The goal of this study was to compare BMI and age-matched African-American and Caucasian women to assess whether traditional biomarkers of insulin resistance, measures of total and regional adiposity, and adipose cell size similarly predict insulin resistance measured by the steady state plasma glucose (SSPG) test between races.

\section{Methods}

Subjects:

Subjects included 34 healthy individuals, 17 African-American and 17 Caucasian women, matched for both age and BMI. This sample size was determined to allow for the observation of significant differences between groups and significant relationships between 
insulin sensitivity and outcome variables at a power of 0.8 , even after adjusting for up to two covariates. Inclusion criteria were BMI $25-35 \mathrm{~kg} / \mathrm{m}^{2}$, age 35 to 65 years old, and generally in good health. Subjects with current or a history of diabetes, cardiovascular disease, liver disease, kidney dysfunction, cancer, bariatric surgery, pregnancy/lactation, chronic inflammatory disease, active psychiatric condition, use of more than 2 alcoholic beverages daily, more than two hours of daily exercise, or significant weight gain or weight loss (>5 lbs.) in the past two months were excluded from participating. All tests were performed at the Center for Translational Research Unit (CTRU) at Stanford University Medical Center. The study was approved by the Stanford University IRB and all subjects gave written, informed consent.

\section{Anthropometric measures and clinical biomarkers of metabolic syndrome:}

Body weight, height, blood pressure, and fasting plasma glucose, total cholesterol, high density lipoprotein (HDL), low density lipoprotein (LDL), and triglycerides were measured at the Clinical Translational Research Unit (CTRU). Waist circumference was measured with arms raised at end expiration, between the iliac crest and the bottom of the rib cage.

\section{Insulin Sensitivity:}

Insulin sensitivity was quantified by the steady-state plasma glucose tolerance test (SSPG), a modification of the insulin suppression test, as previously described and validated $(14,15)$. Briefly, following a 12-hour fast, subjects were infused for 180 minutes with octreotide $\left(0.27 \mu \mathrm{g} / \mathrm{m}^{2} / \mathrm{min}\right)$ to suppress endogenous insulin secretion, and insulin and glucose according to body surface area $\left(25 \mathrm{mU} / \mathrm{m}^{2} / \mathrm{min}\right.$ and $240 \mathrm{mg} / \mathrm{m}^{2} \mathrm{~min}$, respectively). Blood was drawn at 10-minute intervals from 150 to 180 minutes for the measurement of plasma glucose concentrations at steady state when by design, insulin concentrations were similar between subjects $(60 \mathrm{uU} / \mathrm{mL})$. Thus, the mean steady-state plasma glucose (SSPG) concentration in each individual reflects the relative ability of insulin to dispose of a glucose load: the higher the SSPG concentration, the more insulin-resistant the individual.

\section{Regional Fat Distribution:}

Subcutaneous (SAT), visceral (VAT) and thigh adipose tissue volume were quantified using computerized tomography (CT) scans, as previously described (16). Percent VAT and SAT were calculated as the fat in the given depot divided by the total abdominal fat (SAT + VAT) and multiplied by 100. Ratio of VAT to SAT was also calculated. Hepatic fat content was determined by magnetic resonance spectroscopy (MRS) as previously described (17) and expressed as the lipid/H2O ratio. Percent body fat was calculated based on a previously validated formula by Deurenberg and colleagues: $\mathrm{BF} \%=1.2 \times \mathrm{BMI}+0.23 \times$ age $-10.8 \times$ sex -5.4 , where male sex $=1$ and female sex $=0(18)$.

\section{Subcutaneous Fat Biopsy:}

Adipose tissue was excised from all but seven individuals (three African-Americans and four Caucasians who did not consent to the procedure) by periumbilical scalpel biopsy. Adipocytes were isolated using a modified protocol by Rodbell (19) as previously described (20). Adipose tissue was mechanically minced in Modified Eagle's Medium containing 
$0.3 \%$ collagenase for 45 minutes at $37^{\circ} \mathrm{C}$ in a shaking water bath. Digested solution was passed through 450um nylon filter and floating adipocytes were resuspended 3 times in HEPES-Krebs buffer. Stromal vascular cell (SVC) fraction was collected for differentiation. Adipocyte cell size distribution was quantified using the Beckman Multisizer III with a $400 \mu \mathrm{m}$ aperture and effective cell-size range of 20 to $240 \mu \mathrm{m}$. This method generates a frequency histogram plotting the frequency of cells at any given diameter. We have previously shown (20) that the majority of cells follow a Gaussian distribution, the peak of which defines the mean diameter of the mature cell population and is defined as "peak diameter". Additionally, there is a second population of very small cells, defined as "percent small cells," that are separated from the Gaussian by a frequency nadir.

\section{Statistical analysis:}

Although groups were matched for age and BMI, anthropometric and metabolic outcomes were analyzed by ANCOVA analyses (SAS 9.4), adjusting for menopause and BMI for optimal comparison by race without residual confounding. Confirmatory analyses with adjustment for percent body fat instead of BMI were performed. Normality of distribution was performed using Q-Q plots and non-normally distributed variables lot-transformed for normality. The relationships between clinical, metabolic, regional adiposity, and adipose cell variables and SSPG were analyzed using linear regression analyses, separated by racial group with adjustment for BMI and menopause as covariates via stepwise multiple regression analysis to retain maximal statistical power, since in many cases BMI and/or menopause were not independently associated with the dependent variable. Correlations were performed by intent separately by race for descriptive purposes, and formal testing for a race interaction in the relationships between metabolic/adiposity variables and insulin resistance was also performed via construction of linear regression models with interaction terms.

\section{Results}

Table 1 presents clinical, metabolic, regional adiposity, and adipose cell size characteristics of study participants according to race. BMI and age were closely matched. Other comparisons were adjusted statistically for any residual confounding by BMI and menopause. There were no significant differences in blood pressure nor fasting glucose. Insulin sensitivity, as determined by SSPG, was NS different between African-American and Caucasian women. Fasting plasma TG was significantly lower in African-American compared to Caucasian women, while total cholesterol showed only a trend towards lower values in African-American women. Neither HDL- nor LDL-cholesterol differed significantly between groups.

There was only a trend towards higher absolute SAT volume in African-American women, whereas percent SAT volume was significantly higher in African-American compared to Caucasian women. On the contrary, absolute and percent VAT volume, and VAT/SAT ratio were all significantly lower in African-American women. Thigh fat did not differ significantly between races. Intrahepatic fat content was lower in African-American women, 
but p-value was of borderline significance. Adipose cell size distribution measures showed no significant differences between groups.

The relationships between clinical and metabolic determinants of insulin sensitivity measured by SSPG in African-Americans and Caucasians are presented in Figures 1 through 3. Systolic and diastolic blood pressure correlated with SSPG values amongst Caucasian women $(\mathrm{r}=0.59$ and $\mathrm{p}=0.02 ; \mathrm{r}=0.55, \mathrm{p}=0.04)$, although after adjustment for BMI, these relationships lost significance (Figure 1). Fasting plasma glucose was significantly associated with SSPG in both African-American and Caucasian women, even after adjustment for BMI and menopause ( $\mathrm{p}=0.02$ and $\mathrm{p}=0.003$, respectively). While fasting plasma TG was significantly associated with SSPG in both groups, after adjustment for BMI and menopause, these associations remained statistically-significant only in the AfricanAmerican women ( $\mathrm{p}=0.046$ in African-American women versus $\mathrm{p}=0.36$ in Caucasian women). HDL-cholesterol was highly correlated with SSPG in African-American women, even after adjusting for BMI and menopause $(\mathrm{r}=-0.80, \mathrm{p}=0.001)$, but not in Caucasian women. The ratio of TG/HDL correlated with SSPG in African-Americans ( $\mathrm{r}=0.57$, $\mathrm{p}=0.009)$, but did not reach significance in Caucasians (Figure $\mathbf{1} \mathbf{K}$ and $\mathbf{L})$.

Simple metrics of adiposity were evaluated against SSPG values in Figure 2. BMI and percent body fat did not correlate significantly with insulin sensitivity (SSPG) in AfricanAmerican women, but were significant predictors in Caucasian women. Waist circumference was not significantly associated with SSPG in either racial group, although there was a trend in Caucasian women. Figure 3 demonstrates the relationships between measures of regional adiposity and SSPG values by racial group. Absolute VAT, and the ratio of VAT-to-SAT were significantly associated with SSPG in both races, even after adjustment for BMI and menopause. Absolute SAT was not associated with insulin sensitivity in either race but percent SAT correlated inversely $(r=-0.83, p=0.01 ; r=-0.65 p=0.007$, in African-American and Caucasian women, respectively). Thigh fat, after adjustment for BMI and menopause, was significantly inversely associated with SSPG in Caucasians only. Intrahepatic fat was directly associated with SSPG in both groups: $\mathrm{p}=0.04$ in African-American and $\mathrm{p}=0.0001$ in Caucasian women. Intrahepatic fat was also strongly associated with absolute VAT in both groups (African-Americans, r=.69, p0.009; Caucasians, $r=0.78, \mathrm{p}=0.004$ ); data not shown. Formal testing for race interaction with the relationships between these variables and SSPG was negative for all variables. With the exception of peak diameter in Caucasian women, none of the adipose cell size characteristics correlated significantly with SSPG in either race (Figure 4A and B). While peak diameter significantly correlated with SSPG in Caucasian women, a similar relationship was not observed in African-American women. However, upon closer inspection of the data, which appeared to diverge in African-American women according to whether or not they were insulin resistant, a significant relationship between peak diameter and SSPG was observed in the IS subset of African-American women (Figure $4 \mathrm{C}$ ). Formal testing for interaction between insulin sensitivity group and peak cell diameter revealed a significant interaction ( $\mathrm{p}=0.04)$ in African-American, but not in Caucasian women $(\mathrm{p}=0.95)$. 


\section{Discussion}

The results of this study extend a small body of literature addressing the issue of whether African-Americans are more insulin resistant than Caucasians, potentially explaining their higher rates of T2D. Several studies utilizing IVGTT to quantify insulin action suggest that African-Americans are more insulin resistant than Caucasians (4-6). However, the accuracy of the IVGTT in African-Americans may be compromised due to the reliance of insulin concentration in calculations of the sensitivity index. African-Americans exhibit lower insulin clearance rates $(5,10)$ as well as a more robust first phase insulin response following intravenous glucose (12). The minimal model used to assess insulin sensitivity by IVGTT assumes that the concentration of insulin during the first-phase response is equivalent to the amount of insulin secreted (21). Without assessing insulin clearance rates in conjunction with total insulin concentrations in African-Americans insulin resistance can be overestimated in this population (13). Indeed, three studies utilizing the euglycemic hyperinsulinemic clamp method to quantify insulin action showed that African-American men and women were not more insulin resistant than Caucasian counterparts $(13,22,23)$. The current results, which concur with the latter three studies, utilized a whole body measure of insulin-mediated glucose uptake after suppression of endogenous insulin using octreotide. By design, the insulin concentrations are the same in all subjects [19], and thus the plasma glucose during steady state reflects insulin's ability to dispose of a glucose load. Our findings suggest that African-American women are not more insulin resistant that BMImatched Caucasian women, and imply that factors other than insulin resistance may play a role in the disproportionately high rates of T2D that afflict African-American women.

A second important finding in the current study is that in addition to confirming that TG concentrations are lower in African-American than Caucasian women [26,28], TG and HDL-cholesterol and TG/HDL-cholesterol ratio are highly significant predictors of insulin resistance in African-American women. Triglyceride and HDL-cholesterol have been validated as predictors of insulin resistance in a largely Caucasian cohort (24). These markers have been criticized as predictors of insulin resistance in African-Americans (2527), however. Indeed, these variables were not related to insulin resistance, as measured by IVGTT, in a cohort of 98 African-American men and premenopausal women. The current findings contradict these, and strongly suggest that at least in African-American women, despite lower absolute values of TG and higher HDL-cholesterol, these biomarkers predict insulin resistance and formal evaluation for sex and race-specific cut-points are warranted, as previously suggested $(28,29)$.

The current results also demonstrate disparate relationships between adiposity measures and insulin resistance between races. Obesity, typically defined by an individual's BMI, is a well-accepted risk factor for the development of insulin resistance and other metabolic disorders. The current results challenge previous findings that BMI correlates with insulin sensitivity in African-American men and women (26). In the present study, both BMI and percent body fat correlated with insulin resistance in Caucasian but not African-American women. Waist circumference, another accepted marker of metabolic risk (30), which reflects both subcutaneous and visceral fat content, was similarly predictive of insulin resistance only in Caucasian women. Importantly, BMI in our subjects was limited to $25-35 \mathrm{~kg} / \mathrm{m}^{2}$, 
and thus it is possible that among individuals with higher BMIs or waist circumferences, these measures would be predictive of insulin resistance. However, among overweight to moderately obese women, which applies to the majority of Americans, our findings indicate that simple measures of adiposity may not be sufficient in determining risk for insulin resistance and T2D in African-American women.

Contrary to the lack of predictive value of the less specific measures of adiposity, our study highlights the positive predictive value of regional adiposity measures. Several studies, in addition to the current findings, have shown that African-American women tend to have lower VAT compared to Caucasian women $(8,31,32)$. One might question whether VAT is therefore a marker for insulin resistance in this racial group. Our results demonstrate a strong independent positive relationship between VAT, both absolute volume and percent of total abdominal fat, and insulin resistance in African-American as well as in Caucasian women. This suggests a likely lower threshold for VAT content as a predictor of insulin resistance in African-American women. On the contrary, absolute SAT showed no relationship with insulin resistance in either race, and thigh fat was protective in Caucasian but not African-American women. However, the proportion of SAT was inversely associated with insulin resistance in both racial groups. Our findings address contradictory findings in a handful of published studies that have measured regional fat distribution and insulin resistance in African Americans (33-35). In two studies, one conducted in AfricanAmerican children and another in South African women, total and/or subcutaneous fat, but not VAT, was found to predict insulin resistance $(34,35)$ as measured by IVGTT. On the other hand, one study using OGTT as a measure of insulin sensitivity showed that visceral fat and VAT/SAT ratio predicted insulin resistance (33). Ours is the first to utilize a physiologic test for insulin resistance that does not rely on plasma insulin concentrations, but differences in age, menopause, and small sample size in all studies may contribute to the discrepant findings. Larger studies with gold-standard quantitative measures for both insulin sensitivity and regional fat distribution would be helpful.

Similar to visceral fat, hepatic fat content is lower in African-Americans compared to Caucasians $(8,31,33)$. Our study showed a trend towards lower VAT in African-American women, but perhaps more importantly, results showed that VAT and hepatic fat were highly correlated with each other in both African-American and Caucasian women, and significantly associated with insulin resistance in both races. To our knowledge, the present study is the first to demonstrate this relationship in African Americans. These results suggest that hepatic and visceral fat may play a role in the development of insulin resistance in both racial groups.

In order to further examine the potential role of adipose tissue storage in the metabolic health disparities of African-American women, we conducted cell morphology analyses in relationship to insulin resistance. In Caucasian and Pima Indian populations, adipocyte size (hypertrophy) has been noted to predict insulin resistance and the development of T2D better than does BMI [18, 20, 37, 38]. The current study is the first to examine adipocyte size and insulin resistance in African-American women. Interestingly, there was a significant race interaction in the relationship between adipocyte diameter and insulin resistance, with larger cell size predicting insulin resistance only in Caucasian women. We have previously shown 
that impaired adipogenesis characterizes the insulin-resistant state (20), and it is becoming increasingly well accepted that dysfunctional fat storage in the subcutaneous depot may play an important role in determining which individuals develop ectopic fat and metabolic disease as a consequence of excess caloric intake $(17,36)$. While this study is limited by small numbers, it is worth mentioning that the relationship between adipose cell size and insulin resistance showed a bidirectional relationship, with a positive association among the most insulin sensitive African-American but not among the insulin-resistant African-American, whereas the relationship among Caucasian women was similar in both insulin resistant and insulin sensitive. Further exploration of adipocyte function in African-American women is warranted in future studies, and in particular, whether enhanced differentiation in SAT accounts for the lower fat mass in visceral and ectopic depots.

In conclusion, the current results demonstrate that insulin resistance is not greater in African-American women as compared to age and BMI-matched Caucasian women and that while TG is lower in African-American women, TG, HDL-cholesterol, and TG/HDLcholesterol ratio correlate highly with insulin resistance and thus, constitute potential biomarkers. Similarly, while intrahepatic and visceral fat are lower in African-American women, they are directly associated with insulin resistance in both races, whereas less specific indicators of obesity such as BMI, percent body fat and waist circumference are of little predictive value in African-American women with BMI $25-35 \mathrm{~kg} / \mathrm{m}^{2}$. The observed differences in the relationships between regional fat and plasma lipid concentrations with insulin resistance likely rest in race differences in adipocyte function, as reflected by the race-interaction noted in the relationship between adipocyte hypertrophy and insulin resistance. Future research should further investigate the molecular basis for the findings presented in this study to better understand race differences in the biological basis for obesity-associated insulin resistance.

\section{Acknowledgements}

This study was supported by grants from the American Diabetes Association (1-11-CT-35), NIH/NIDDK (1RO01 DK090436) and the Building Interdisciplinary Research Careers in Women's Health award (K12 HD051958) funded by the National Institute of Child Health and Human Development (NICHD), Office of Research on Women's Health, Office of Dietary Supplements, and that National Institute of Aging.

\section{Abbreviations}

$\begin{array}{ll}\text { SSPG } & \text { Steady state plasmas glucose } \\ \text { HDL } & \text { high density lipoprotein } \\ \text { LDL } & \text { low density lipoprotein } \\ \text { TG } & \text { triglycerides } \\ \text { SAT } & \text { subcutaneous adipose tissue } \\ \text { VAT } & \text { visceral adipose tissue }\end{array}$




\section{References}

1. American Diabetes A. National Diabetes Statistics Report. http://wwwdiabetesorg/diabetes-basics/ statistics/. 2014.

2. Centers for Disease Control aP. Differences in Prevalence of Obesity Among Black, White, and Hispanic Adults --- United States, 2006--2008. https://wwwcdcgov/mmwr/preview/mmwrhtml/ mm5827a2htm. 2009.

3. Zhang Q, Wang Y, Huang ES. Changes in racial/ethnic disparities in the prevalence of Type 2 diabetes by obesity level among US adults. Ethn Health. 2009;14(5):439-57. [PubMed: 19360513]

4. Chow CC, Periwal V, Csako G, Ricks M, Courville AB, Miller BV 3rd, et al. Higher acute insulin response to glucose may determine greater free fatty acid clearance in African-American women. $\mathbf{J}$ Clin Endocrinol Metab. 2011;96(8):2456-63. [PubMed: 21593106]

5. Haffner SM, D’Agostino R, Saad MF, Rewers M, Mykkanen L, Selby J, et al. Increased insulin resistance and insulin secretion in nondiabetic African-Americans and Hispanics compared with non-Hispanic whites. The Insulin Resistance Atherosclerosis Study. Diabetes. 1996;45(6):742-8. [PubMed: 8635647]

6. Hyatt TC, Phadke RP, Hunter GR, Bush NC, Munoz AJ, Gower BA. Insulin sensitivity in AfricanAmerican and white women: association with inflammation. Obesity (Silver Spring). 2009;17(2): 276-82. [PubMed: 19039315]

7. Robbins JM, Vaccarino V, Zhang H, Kasl SV. Socioeconomic status and type 2 diabetes in African American and non-Hispanic white women and men: evidence from the Third National Health and Nutrition Examination Survey. Am J Public Health. 2001;91(1):76-83. [PubMed: 11189829]

8. Lovejoy JC, de la Bretonne JA, Klemperer M, Tulley R. Abdominal fat distribution and metabolic risk factors: effects of race. Metabolism. 1996;45(9):1119-24. [PubMed: 8781299]

9. Perry AC, Applegate EB, Jackson ML, Deprima S, Goldberg RB, Ross R, et al. Racial differences in visceral adipose tissue but not anthropometric markers of health-related variables. J Appl Physiol (1985). 2000;89(2):636-43. [PubMed: 10926648]

10. Wang L, Sacks FM, Furtado JD, Ricks M, Courville AB, Sumner AE. Racial differences between African-American and white women in insulin resistance and visceral adiposity are associated with differences in apoCIII containing apoAI and apoB lipoproteins. Nutr Metab (Lond). 2014;11(1):56. [PubMed: 25553059]

11. Lee CC, Haffner SM, Wagenknecht LE, Lorenzo C, Norris JM, Bergman RN, et al. Insulin clearance and the incidence of type 2 diabetes in Hispanics and African Americans: the IRAS Family Study. Diabetes Care. 2013;36(4):901-7. [PubMed: 23223351]

12. Arslanian S, Suprasongsin C. Differences in the in vivo insulin secretion and sensitivity of healthy black versus white adolescents. J Pediatr. 1996;129(3):440-3. [PubMed: 8804335]

13. Pisprasert V, Ingram KH, Lopez-Davila MF, Munoz AJ, Garvey WT. Limitations in the use of indices using glucose and insulin levels to predict insulin sensitivity: impact of race and gender and superiority of the indices derived from oral glucose tolerance test in African Americans.

Diabetes Care. 2013;36(4):845-53. [PubMed: 23223406]

14. Greenfield MS, Doberne L, Kraemer F, Tobey T, Reaven G. Assessment of insulin resistance with the insulin suppression test and the euglycemic clamp. Diabetes. 1981;30(5):387-92. [PubMed: 7014307]

15. Pei D, Jones CN, Bhargava R, Chen YD, Reaven GM. Evaluation of octreotide to assess insulinmediated glucose disposal by the insulin suppression test. Diabetologia. 1994;37(8):843-5. [PubMed: 7988789]

16. McLaughlin T, Lamendola C, Liu A, Abbasi F. Preferential fat deposition in subcutaneous versus visceral depots is associated with insulin sensitivity. J Clin Endocrinol Metab. 2011;96(11):E1756-60. [PubMed: 21865361]

17. McLaughlin T, Craig C, Liu LF, Perelman D, Allister C, Spielman D, et al. Adipose Cell Size and Regional Fat Deposition as Predictors of Metabolic Response to Overfeeding in Insulin-Resistant and Insulin-Sensitive Humans. Diabetes. 2016;65(5):1245-54. [PubMed: 26884438]

18. Deurenberg P, Weststrate JA, Seidell JC. Body mass index as a measure of body fatness: age- and sex-specific prediction formulas. Br J Nutr. 1991;65(2):105-14. [PubMed: 2043597] 
19. Aarsland A, Wolfe RR. Hepatic secretion of VLDL fatty acids during stimulated lipogenesis in men. J Lipid Res. 1998;39(6):1280-6. [PubMed: 9643360]

20. McLaughlin T, Sherman A, Tsao P, Gonzalez O, Yee G, Lamendola C, et al. Enhanced proportion of small adipose cells in insulin-resistant vs insulin-sensitive obese individuals implicates impaired adipogenesis. Diabetologia. 2007;50(8):1707-15. [PubMed: 17549449]

21. Bergman RN, Phillips LS, Cobelli C. Physiologic evaluation of factors controlling glucose tolerance in man: measurement of insulin sensitivity and beta-cell glucose sensitivity from the response to intravenous glucose. J Clin Invest. 1981;68(6):1456-67. [PubMed: 7033284]

22. Hannon TS, Bacha F, Lin Y, Arslanian SA. Hyperinsulinemia in African-American adolescents compared with their American white peers despite similar insulin sensitivity: a reflection of upregulated beta-cell function? Diabetes Care. 2008;31(7):1445-7. [PubMed: 18417751]

23. Lee $\mathrm{S}$, Guerra N, Arslanian S. Skeletal muscle lipid content and insulin sensitivity in black versus white obese adolescents: is there a race differential? J Clin Endocrinol Metab. 2010;95(5):242632. [PubMed: 20219892]

24. McLaughlin T, Abbasi F, Cheal K, Chu J, Lamendola C, Reaven G. Use of metabolic markers to identify overweight individuals who are insulin resistant. Annals of Internal Medicine. 2003;139(10):802-9. [PubMed: 14623617]

25. Sumner AE. "Half the dsylipidemia of insulin resistance" is the dyslipidemia [corrected] of insulinresistant Blacks. Ethn Dis. 2009;19(4):462-5. [PubMed: 20073149]

26. Sumner AE, Vega GL, Genovese DJ, Finley KB, Bergman RN, Boston RC. Normal triglyceride levels despite insulin resistance in African Americans: role of lipoprotein lipase. Metabolism. 2005;54(7):902-9. [PubMed: 15988699]

27. Kim-Dorner SJ, Deuster PA, Zeno SA, Remaley AT, Poth M. Should triglycerides and the triglycerides to high-density lipoprotein cholesterol ratio be used as surrogates for insulin resistance? Metabolism. 2010;59(2):299-304. [PubMed: 19796777]

28. Li C, Ford ES, Meng YX, Mokdad AH, Reaven GM. Does the association of the triglyceride to high-density lipoprotein cholesterol ratio with fasting serum insulin differ by race/ethnicity? Cardiovasc Diabetol. 2008;7:4. [PubMed: 18307789]

29. Stein E, Kushner H, Gidding S, Falkner B. Plasma lipid concentrations in nondiabetic African American adults: associations with insulin resistance and the metabolic syndrome. Metabolism. 2007;56(7):954-60. [PubMed: 17570258]

30. Klein S, Allison DB, Heymsfield SB, Kelley DE, Leibel RL, Nonas C, et al. Waist circumference and cardiometabolic risk: a consensus statement from Shaping America's Health: Association for Weight Management and Obesity Prevention; NAASO, The Obesity Society; the American Society for Nutrition; and the American Diabetes Association. Am J Clin Nutr. 2007;85(5):1197202. [PubMed: 17490953]

31. Goran MI, Nagy TR, Treuth MS, Trowbridge C, Dezenberg C, McGloin A, et al. Visceral fat in white and African American prepubertal children. Am J Clin Nutr. 1997;65(6):1703-8. [PubMed: 9174463]

32. Weinsier RL, Hunter GR, Gower BA, Schutz Y, Darnell BE, Zuckerman PA. Body fat distribution in white and black women: different patterns of intraabdominal and subcutaneous abdominal adipose tissue utilization with weight loss. Am J Clin Nutr. 2001;74(5):631-6. [PubMed: 11684531]

33. Albu JB, Murphy L, Frager DH, Johnson JA, Pi-Sunyer FX. Visceral fat and race-dependent health risks in obese nondiabetic premenopausal women. Diabetes. 1997;46(3):456-62. [PubMed: 9032103]

34. Goedecke JH, Levitt NS, Lambert EV, Utzschneider KM, Faulenbach MV, Dave JA, et al. Differential effects of abdominal adipose tissue distribution on insulin sensitivity in black and white South African women. Obesity (Silver Spring). 2009;17(8):1506-12. [PubMed: 19300428]

35. Goran MI, Bergman RN, Gower BA. Influence of total vs. visceral fat on insulin action and secretion in African American and white children. Obes Res. 2001;9(8):423-31. [PubMed: 11500522]

36. Vidal-Puig A Adipose tissue expandability, lipotoxicity and the metabolic syndrome. Endocrinol Nutr. 2013;60 Suppl 1:39-43. [PubMed: 24490226] 
A

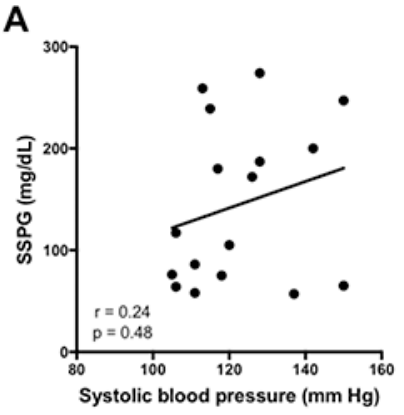

C

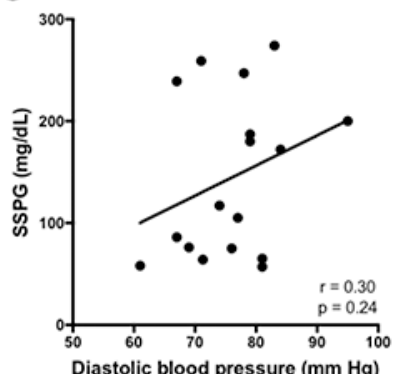

E

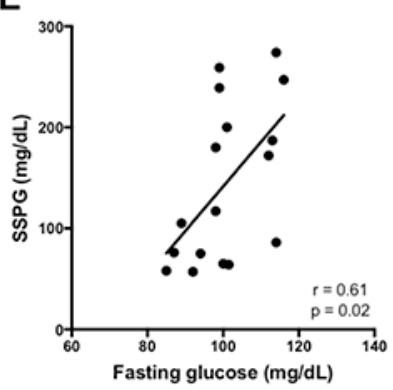

B

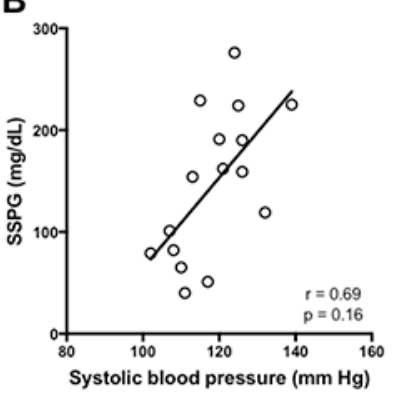

D

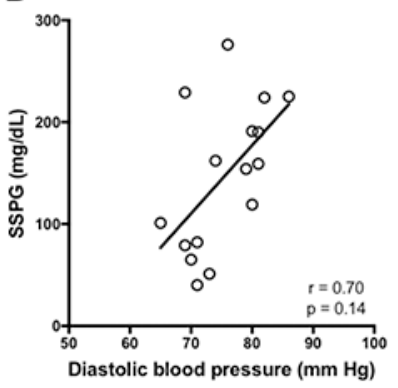

$\mathbf{F}$

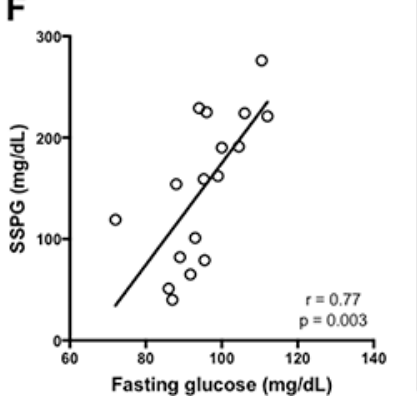

G

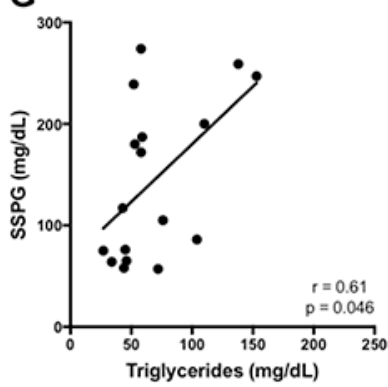

Triglycerides $(\mathrm{mg} / \mathrm{dL})$

I

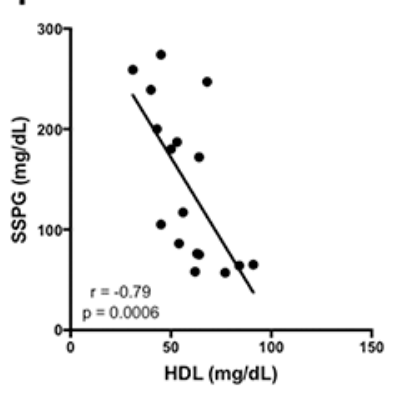

$\mathbf{K}$

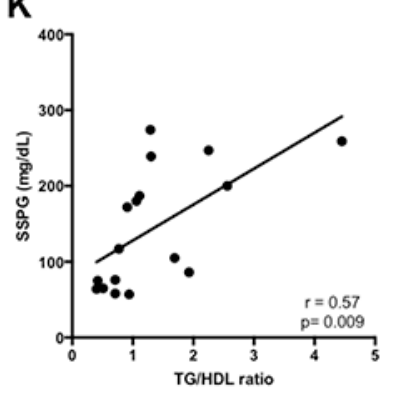

H

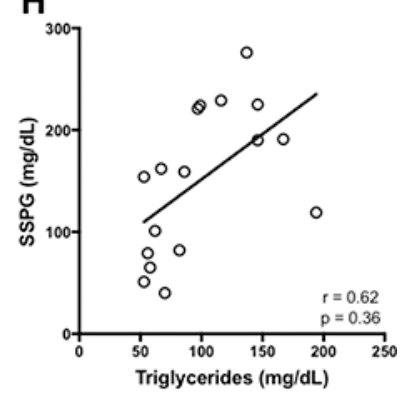

J

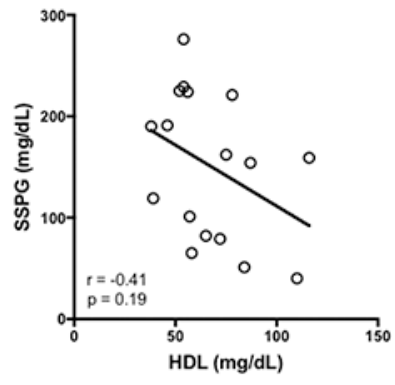

L

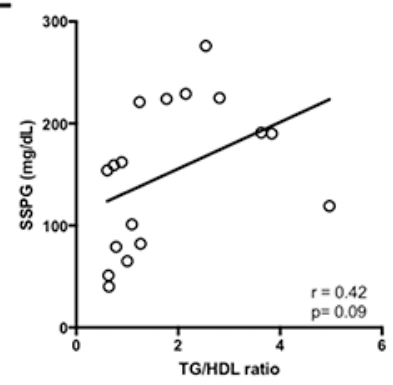

Figure 1:

Relationships between insulin sensitivity and metabolic characteristics in African-American women compared to Caucasian women. African-Americans shown on left ( ). Caucasians shown on right $(\bigcirc)$. Data shown are based on stepwise multiple linear regression analyses with adjustments for BMI and menopausal status. 
A

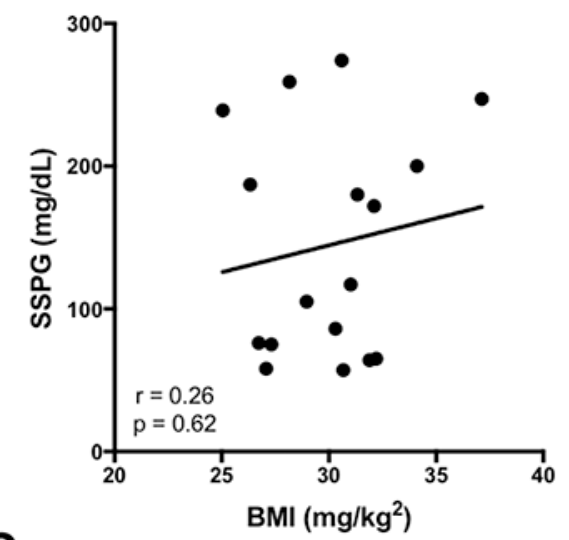

C

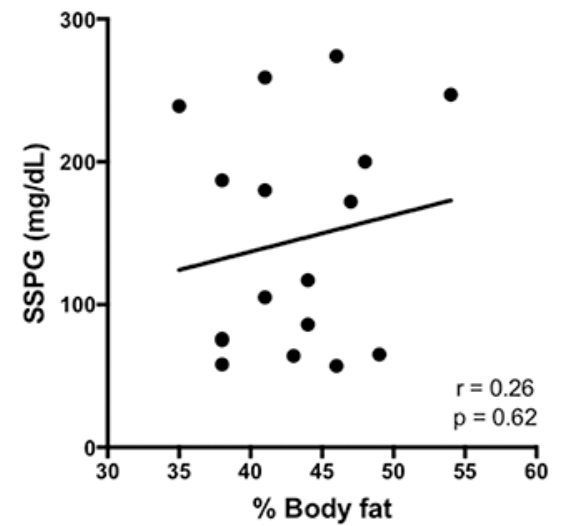

E

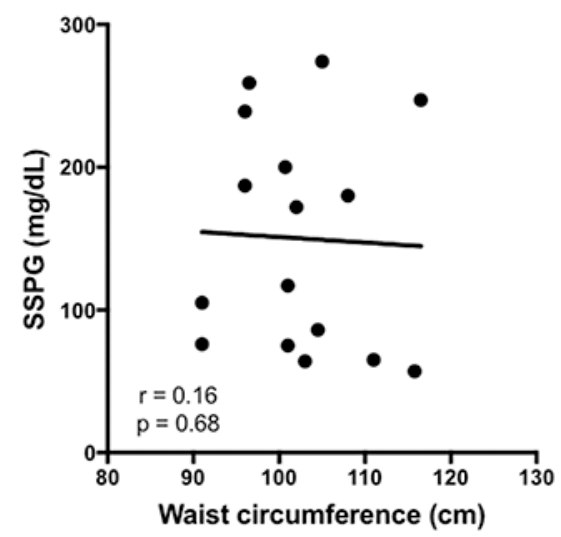

B

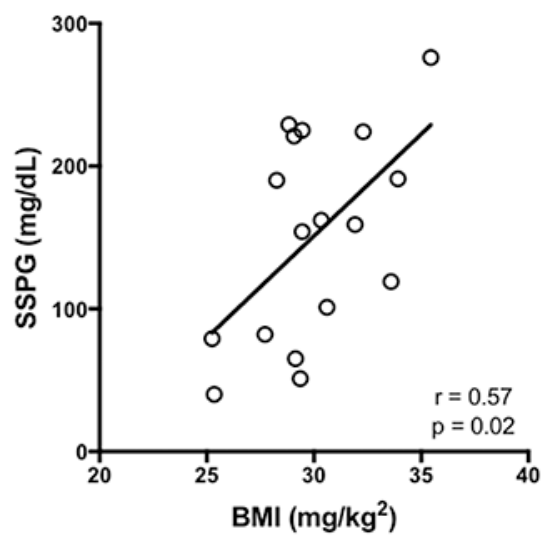

D

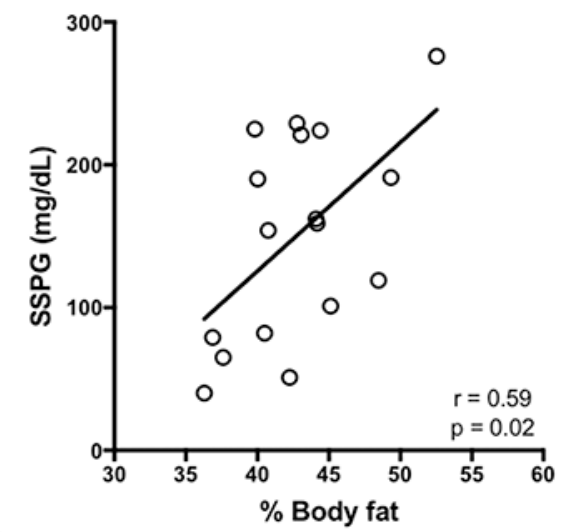

$\mathbf{F}$

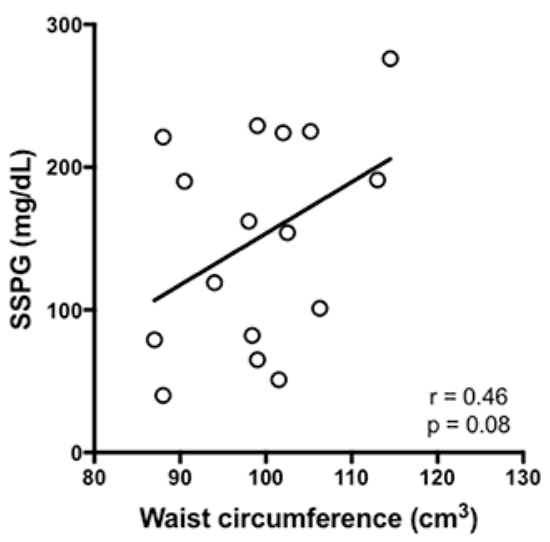

Figure 2:

Relationships between insulin sensitivity and simple measures of obesity in AfricanAmerican women compared to Caucasian women. African-Americans shown on left (). Caucasians shown on right $(\bigcirc)$. Data shown are based on stepwise multiple linear regression analyses with adjustments for BMI and menopausal status. 

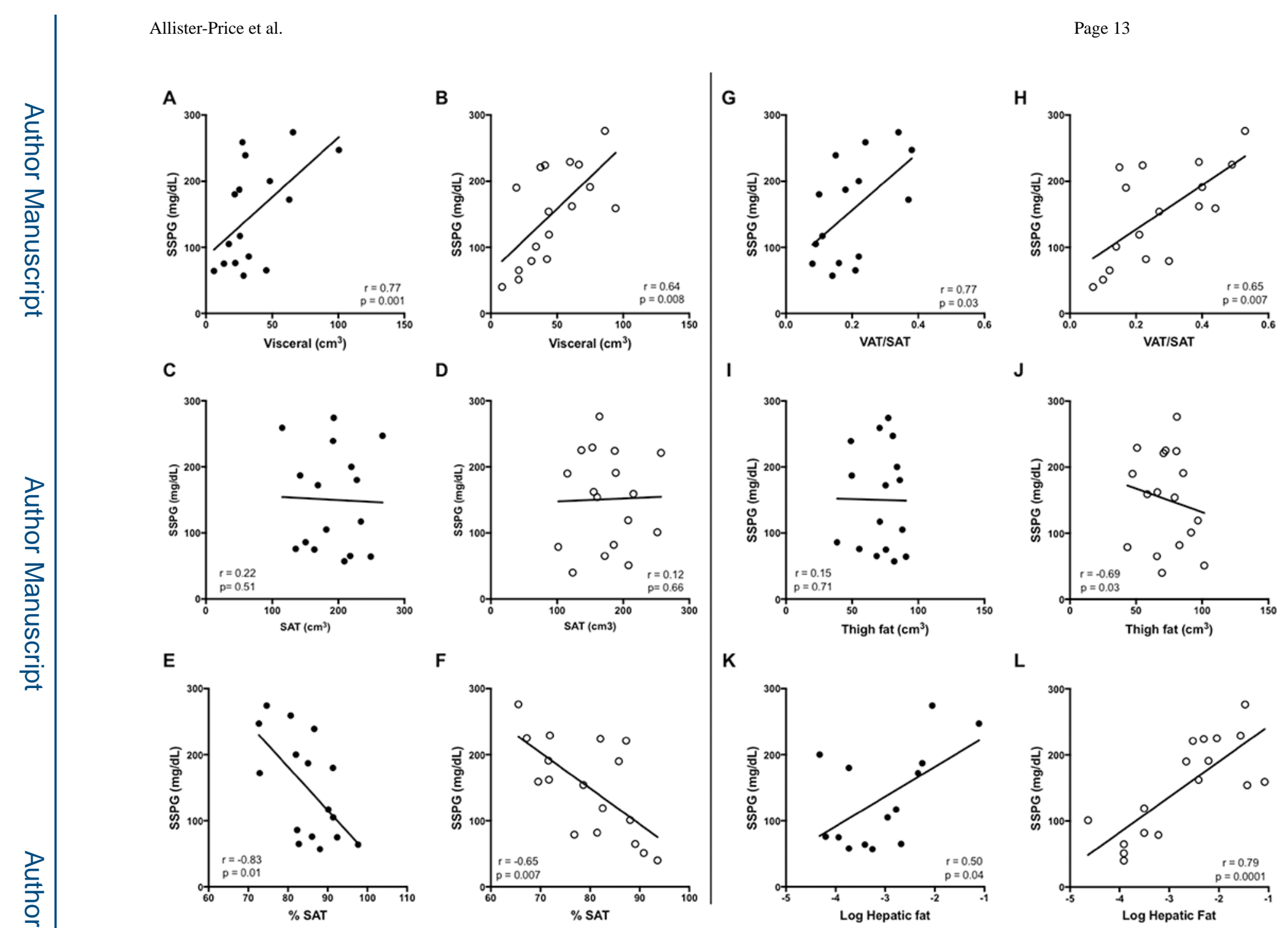

L

Figure 3:

Relationships between insulin sensitivity and measures of fat distribution in AfricanAmerican women compared to Caucasian women. African-Americans shown on left ( $)$. Caucasians shown on right $(\bigcirc)$. Data shown are based on stepwise multiple linear regression analyses with adjustments for BMI and menopausal status. 

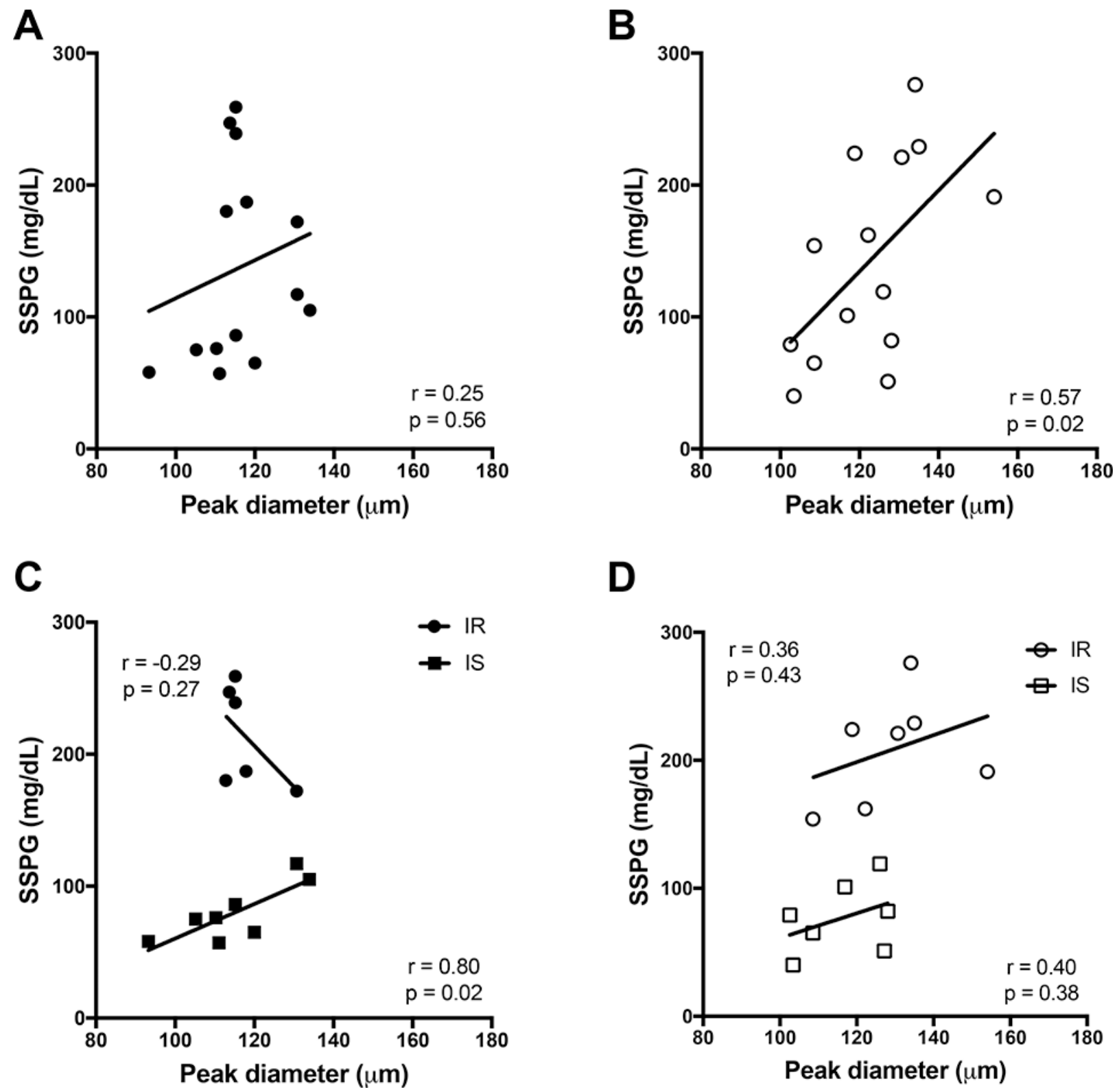

Figure 4:

Peak diameter versus SSPG in African-American and Caucasian women. A) AfricanAmerican women, solid circles $(n=14)$; B) Caucasian women, open circles $(n=14)$; Data shown are based on stepwise multiple linear regression analyses with adjustments for BMI and menopausal status. C) African-American women categorized by insulin sensitivity; D) Caucasian women categorized by insulin sensitivity. Insulin sensitive individuals are identified by squares. Insulin resistant individuals are identified by circles. Data shown based on linear regression analysis separated by insulin sensitivity. 
Table 1:

Clinical and metabolic subject characteristics

\begin{tabular}{|c|c|c|c|}
\hline & African-American $(n=17)$ & Caucasian $(n=17)$ & $p^{*}$ \\
\hline Age (yrs) & $53.0 \pm 7.7$ & $52.9 \pm 7.4$ & 0.93 \\
\hline Premenopausal/postmenopausal & $6 / 11$ & $9 / 8$ & 0.38 \\
\hline BMI $\left(\mathrm{kg} / \mathrm{m}^{2}\right)$ & $30.1 \pm 0.7$ & $29.9 \pm 2.7$ & 0.93 \\
\hline Waist circumference (cm) & $102.4 \pm 7.7$ & $98.5 \pm 8.0$ & 0.18 \\
\hline \multicolumn{4}{|l|}{ Blood pressure (mm Hg) } \\
\hline Systolic & $122.5 \pm 14.8$ & $120.8 \pm 11.1$ & 0.37 \\
\hline Diastolic & $76.1 \pm 8.0$ & $77 \pm 5.7$ & 0.96 \\
\hline Fasting glucose $(\mathbf{m g} / \mathbf{d L})$ & $100.7 \pm 9.9$ & $95.8 \pm 10.0$ & 0.18 \\
\hline $\operatorname{SSPG}(\mathrm{mg} / \mathrm{dL})$ & $144.8 \pm 78.8$ & $157.5 \pm 72.5$ & 0.65 \\
\hline Total cholesterol (mg/dL) & $175.9 \pm 28.4$ & $196.2 \pm 27.7$ & 0.07 \\
\hline Plasma TG (mg/dL) & $68.9 \pm 36.3$ & $104.5 \pm 44.6$ & 0.01 \\
\hline HDL (mg/dL) & $58.2 \pm 15.9$ & $66.3 \pm 23.1$ & 0.22 \\
\hline LDL (mg/dL) & $103.8 \pm 31.7$ & $113.5 \pm 30.4$ & 0.48 \\
\hline TG/HDL ratio & $1.4 \pm 1.0$ & $1.8 \pm 1.3$ & 0.19 \\
\hline Subcutaneous adipose tissue $\left(\mathrm{cm}^{3}\right)$ & $191.7 \pm 43.5$ & $165.3 \pm 42.4$ & 0.10 \\
\hline \%Subcutaneous fat & $84.8 \pm 7.2$ & $77.7 \pm 9.2$ & $0.005^{*}$ \\
\hline Visceral adipose tissue $\left(\mathrm{cm}^{3}\right)$ & $35.8 \pm 23.9$ & $48.3 \pm 23.8$ & $0.02^{*}$ \\
\hline$\%$ Visceral fat & $15.2 \pm 7.2$ & $22.3 \pm 9.2$ & $0.005^{*}$ \\
\hline VAT/SAT ratio & $0.2 \pm 0.1$ & $0.3 \pm 0.2$ & $0.01 *$ \\
\hline Thigh fat $\left(\mathrm{cm}^{3}\right)$ & $71.4 \pm 15.5$ & $70.8 \pm 16.0$ & 0.9 \\
\hline Hepatic fat $\left(\mathrm{cm}^{3}\right)$ & $0.07 \pm 0.8$ & $0.16 \pm 0.18$ & $0.07 \xi$ \\
\hline \multicolumn{4}{|l|}{ Adipocyte cell size } \\
\hline Nadir $(\mu \mathrm{m})$ & $76.1 \pm 6.8$ & $75.4 \pm 12.3$ & 0.72 \\
\hline$\%$ Small & $58.7 \pm 14.1$ & $55.8 \pm 14.1$ & 0.53 \\
\hline Peak diameter $(\mu \mathrm{m})$ & $116.1 \pm 10.7$ & $121.1 \pm 15.8$ & 0.12 \\
\hline
\end{tabular}

